\begin{tabular}{|c|l|}
\hline Title & $\begin{array}{l}\text { Sensitivity and uncertainty analysis for reactor stable period induced by positive reactivity using one point adjoint } \\
\text { kinetics equation }\end{array}$ \\
\hline Author(s) & Chiba, Go; Tsuji, Masashi; Narabay ashi, Tadashi \\
\hline Citation & $\begin{array}{l}\text { Journal of nuclear science and technology, 50(12), 1150-1160 } \\
\text { https://doi.org/10.1080/00223131.2013.838332 }\end{array}$ \\
\hline Issue Date & 2013-12-01 \\
\hline Doc URL & http://hdl.handle.net/2115/57482 \\
\hline Rights & $\begin{array}{l}\text { Thisis an A uthor's A ccepted Manuscript of an article published in Journal of Nuclear Science and Technology } \\
\text { (Published in Dec.2013), available online at: http:/www.tandfonline.com/doi /pdf/10.1080/00223131.2013.838332. }\end{array}$ \\
\hline Type & article (author version) \\
\hline File Information & Sensitivity and uncertainty analysis for reactor.pdf \\
\hline
\end{tabular}

Instructions for use 


\title{
Sensitivity and uncertainty analysis for reactor stable period induced by positive reactivity using one-point adjoint kinetics equation
}

\author{
Go CHIBA* $^{* a}$, Masashi TSUJI ${ }^{a}$ and Tadashi NARABAYASHI ${ }^{a}$ \\ ${ }^{a}$ Graduate School of Engineering, Hokkaido University, Sapporo 060-8628, Japan
}

In order to better predict kinetic behavior of a nuclear fission reactor, improvement of delayed neutron parameters is essential. Since it is required to establish a path from the microscopic nuclear data to the macroscopic delayed neutron parameters for the improvement, the present paper identifies important nuclear data for reactor kinetics. Sensitivities of the reactor stable period, which describes reactor kinetic behavior, to microscopic nuclear data such as independent fission yields, decay constants and decay branching ratios are calculated efficiently by using the adjoint kinetics equation. Furthermore, nuclide-wise and nuclear data-wise uncertainties of the reactor stable period are quantified using the variance data given in the nuclear data file, and the nuclear data which require further improvement are identified.

The results obtained through the present study are quite helpful, and can be a driving force of further nuclear physic studies.

KEYWORDS: reactor kinetics, reactor stable period, one-point adjoint kinetics equations, 


\section{Introduction}

The reactor stable period, which is a time constant describing kinetic behavior of a nuclear fission reactor, is quite an important measurable parameter. By putting a value of the reactor stable period into the well-known inhour equation, we can deduce a reactivity of a reactor and quantify how far the reactor state is from a critical state, i.e., a balanced state from a view point of fission chain reactions. Even though a very small fraction of fission neutrons is emitted from fission product nuclides with some of time delay, these neutrons, called as delayed neutrons, play a crucial role in fission chain reactions especially below a prompt critical state.

The inhour equation which gives a relation between a reactivity and a reactor stable period includes some physical parameters such as fission yields and decay constants of delayed neutron precursors. Since there are so many fission product nuclides which or whose daughter nuclides emit delayed neutrons after radioactive decay, these fission product nuclides are grouped to a few families in accordance with their decay constants, and the grouped delayed neutron precursors are treated in the inhour equation. From the very old day when the nuclear energy utilization began, the delayed neutron precursors have been grouped into the six families and the treatment of the six-group delayed neutron precursors has been widely applied[1, 2]. It should be noted that the eight-group treatment was also proposed about ten years ago in order to improve the prediction of reactor behavior under a large negative reactivity insertion, and the JEFF-3.1 nuclear data library adopts the eight-group parameters[3].

A challenge by Brady and England made in 1989 have shown us a new possibility to

${ }^{*}$ Corresponding author, E-mail: go_chiba@eng.hokudai.ac.jp 
improve the delayed neutron-related data[4]. They have evaluated the delayed neutronrelated physical data for each fission product nuclide with the measurement data and model calculation results, have performed a summation calculation using the evaluated delayed neutron data and have fitted the six-group constants to the results of the summation calculation. This challenge connects the microscopic nuclear data with the macroscopic grouped delayed neutron data, and it gives us a possibility to better understand reactor kinetic behavior through studying the fundamental nuclear physics. The delayed neutron parameters evaluated by Brady and England have been adopted in the ENDF/B-VI nuclear data library and also the latest version of the ENDF/B library, ENDF/B-VII.1.

Though the work done by Brady and England has played quite an important role in this field, a problem of their evaluated parameters has been pointed out by some authors $[5,6]$. While the widely used delayed neutron parameters evaluated by Keepin yields consistent results with experimental data for the reactor kinetic behavior, some deficiencies have been observed when Brady and England's data are used. When the same value of the reactor stable period is given, a reactivity value deduced with Brady and England's delayed neutron parameters is over $10 \%$ lower than that deduced with Keepin's data for the uranium-235 thermal fission. This difference is significantly large from a view point of actual application.

A study done by Oyamatsu has addressed this issue[7]. He has pointed out that the iodine-137 contribution may be responsible for the difference between Keepin's evaluated parameters and Brady\&England's parameters, and also has quantified a nuclide-wise contribution to the reactor stable period $T$ at a limit of $T \rightarrow \infty$. He has identified some delayed neutron precursors which have strong influence on the reactor kinetic behavior.

More recently, the present authors have carried out a sensitivity analysis[8] for the 
reactor stable period in order to identify important delayed neutron-related nuclear data with a different approach to Oyamatsu's work. They have used an adjoint equation of the one-point reactor kinetics equation and have quantified importance of fission product nuclides for the reactor kinetics. Since their approach is not restricted to a limit of $T \rightarrow \infty$ as Oyamatsu's work, they have obtained information which complements Oyamatsu's preceding work. They have also calculated sensitivities of the reactor stable period to the delayed neutron-related nuclear data using several different nuclear data files, and have shown that there are large differences in the sensitivities among the different nuclear data files even though currently evaluated nuclear data files are used.

While there have been several works on the adjoint reactor kinetics equation, its application to the one-point equation with the explicit fission product treatment is new as far as authors' knowledge. Here we cite only two papers on the adjoint reactor kinetics equation. $[9,10]$ The paper written by van Rooijen and Lathouwers presents a sensitivity analysis for six-group delayed neutron data in multi-dimensional problems.

Once sensitivities of the reactor stable period to nuclear data are obtained, uncertainty propagation calculations from nuclear data to the reactor stable period can be easily done. In the present study, the uncertainty of the reactor stable period is quantified using the sensitivities calculated with the manner proposed in the previous study and uncertainty information given in the nuclear data file. By quantifying nuclide-wise and nuclear datawise uncertainties in the reactor stable period, delayed neutron data which should be more precisely measured and/or more accurately modeled can be identified. Such an information can be a driving force of further nuclear physic studies.

Due to the page limitation, reference [8] gives the very brief theoretical background of sensitivity calculations with the adjoint kinetics equation and limited numerical examples 
only for the uranium-235 and plutonium-239 thermal fission cases. In order to complement the previous paper, the present paper includes very detail description of the theoretical background and gives numerical results for the plutonium-239 and uranium-238 fast fission cases in addition to the thermal fission cases.

The present paper is organized as follows. Section 2 explains the one-point reactor kinetics equation and its adjoint form, and a theory of sensitivity calculations using the adjoint function. In Sec. 3, a numerical procedure and used nuclear data are described, and we will show sensitivities of reactor stable periods to fission yields and decay constants of fission product nuclides, and nuclide-wise and nuclear data-wise uncertainties in the reactor stable period for various types of fissioning systems. These are summarized in Sec. 4.

\section{Theory}

\subsection{One-point reactor kinetics equation and its adjoint form}

The theory of the adjoint kinetics equation has been well established. Here it is briefly described for readers' benefits.

Let us consider a system to which a constant (time-independent) reactivity $\rho$ is added. The one-point reactor kinetics equations are written as follows:

$$
\begin{aligned}
\frac{d n(t)}{d t} & =\frac{\rho-\beta}{\Lambda} n(t)+\sum_{i=1}^{N} \lambda_{i} C_{i}(t) P n_{i}, \\
\frac{d C_{i}(t)}{d t} & =\frac{y_{i}}{\nu \Lambda} n(t)-\lambda_{i} C_{i}(t)+\sum_{j=1}^{N} \lambda_{j} C_{j}(t) P_{j i}, \quad(i=1, \ldots, N),
\end{aligned}
$$

where $n(t)$ is the number of neutrons at time $t, \Lambda$ is the neutron generation time, $\nu$ is the averaged number of neutrons emitted by a fission reaction, and $N$ denotes the total 
number of considered fission product nuclides. Parameters $C_{i}, P n_{i}, y_{i}, \lambda_{i}$ denote a number density, a neutron emission probability per a decay, a fission yield and a decay constant of a fission product nuclide $i . \beta$ is a delayed neutron fraction and is defined as

$$
\beta=\frac{\sum_{i=1}^{N} \bar{y}_{i} P n_{i}}{\nu}=\frac{\sum_{i=1}^{N} y_{i} D_{i}}{\nu},
$$

where $\bar{y}_{i}$ is the cumulative fission yield and $D_{i}$ the total number of emitted delayed neutrons per a generation of nuclide $i$ : total number of delayed neutrons emitted by the nuclide $i$ and its all daughter nuclides. $P_{j i}$ gives a probability that nuclide $i$ is produced by a decay of nuclide $j$. Equations (1) and (2) consider delayed neutron emissions from not only precursors which are generated directly by a fission reaction but also those generated by a radioactive decay of a parent fission product nuclide. This effect is referred to as a decay chain effect in the present paper. The importance of the decay chain effect has been pointed out in the previous studies.[11, 12]

If we neglect the decay chain effect and assume that all the delayed neutron precursors are generated instantaneously by a fission reaction, the one-point kinetics equations can be simply written as follows:

$$
\begin{aligned}
\frac{d n(t)}{d t} & =\frac{\rho-\beta}{\Lambda} n(t)+\sum_{i=1}^{N} \lambda_{i} C_{i}(t) P n_{i}, \\
\frac{d C_{i}(t)}{d t} & =\frac{\bar{y}_{i}}{\nu \Lambda} n(t)-\lambda_{i} C_{i}(t), \quad(i=1, \ldots, N) .
\end{aligned}
$$

In this case, relation between a reactivity $\rho$ and a reactor stable period $T$ can be easily derived as

$$
\rho=\frac{l}{T+l}+\frac{T}{T+l} \sum_{i=1}^{N} \frac{\beta_{i}}{1+\lambda_{i} T},
$$

where $l$ denotes a prompt neutron life time and $\beta_{i}=\bar{y}_{i} P n_{i} / \nu$.

For the purpose of the present study, the one-point kinetics equations (1) and (2) are 
rewritten as follows:

$$
\begin{aligned}
\frac{d n(t)}{d t} & =\frac{k_{p}-1}{l} n(t)+\sum_{i=1}^{N} \lambda_{i} C_{i}(t) P n_{i}, \\
\frac{d C_{i}(t)}{d t} & =\frac{k_{p}}{l} \frac{y_{i}}{\nu_{p}} n(t)-\lambda_{i} C_{i}(t)+\sum_{j=1}^{N} \lambda_{j} C_{j}(t) P_{j i}, \quad(i=1, \ldots, N),
\end{aligned}
$$

where $k_{p}$ is a prompt neutron multiplication factor which neglects delayed neutron contributions to a fission chain reaction and $\nu_{p}$ is the averaged number of prompt neutrons emitted by a fission reaction. For simplicity, these equations are written in a matrix form as

$$
\frac{d \boldsymbol{y}}{d t}=\boldsymbol{A y}
$$

where $\boldsymbol{y}=\left(n, C_{1}, C_{2}, \ldots, C_{N}\right)^{T}$. An adjoint equation to Equation (9) can be easily derived as

$$
-\frac{d \boldsymbol{y}^{\dagger}}{d t}=\boldsymbol{A}^{T} \boldsymbol{y}^{\dagger}
$$

where $\boldsymbol{y}^{\dagger}=\left(n^{\dagger}, C_{1}^{\dagger}, C_{2}^{\dagger}, \ldots, C_{N}^{\dagger}\right)^{T}$. Its explicit form is given as

$$
\begin{aligned}
-\frac{d n^{\dagger}(t)}{d t} & =\frac{k_{p}-1}{l} n^{\dagger}(t)+\sum_{i=1}^{N} \frac{k_{p}}{l} \frac{y_{i}}{\nu_{p}} C_{i}^{\dagger}(t) \\
-\frac{d C_{i}^{\dagger}(t)}{d t} & =\lambda_{i} P n_{i} n^{\dagger}(t)-\lambda_{i} C_{i}^{\dagger}(t)+\sum_{j=1}^{N} \lambda_{i} C_{j}^{\dagger}(t) P_{i j}, \quad(i=1, \ldots, N) .
\end{aligned}
$$

\subsection{Sensitivity of reactor stable period to delayed neutron data}

A reactor stable period $T$ can be defined from the numbers of neutrons at two different time points $t_{1}$ and $t_{2}=t_{1}+\Delta t$ in an asymptotic state as

$$
T=\frac{\Delta t}{\ln \left\{n\left(t_{2}\right) / n\left(t_{1}\right)\right\}} .
$$


A derivative of $T$ to a delayed neutron data $p$, such as a fission yield and a decay constant, can be calculated as

$$
\frac{\partial T}{\partial p}=\frac{\partial T}{\partial n\left(t_{1}\right)} \frac{\partial n\left(t_{1}\right)}{\partial p}+\frac{\partial T}{\partial n\left(t_{2}\right)} \frac{\partial n\left(t_{2}\right)}{\partial p} .
$$

In order to obtain $\frac{\partial T}{\partial p}$, it is required to calculate $\frac{\partial n\left(t_{1}\right)}{\partial p}$ and $\frac{\partial n\left(t_{2}\right)}{\partial p}$. These derivatives can be obtained numerically by taking a difference of two numerical results: a base result and a perturbed result. This approach, however, requires so many cases of numerical calculations. In the present study, we propose to use the adjoint function $\boldsymbol{y}^{\dagger}$ to calculate these derivatives as follows.

By differentiating the both sides of Equation (9) by $p$, we can obtain

$$
\frac{d}{d t}\left(\frac{d \boldsymbol{y}}{d p}\right)=\frac{d \boldsymbol{A}}{d p} \boldsymbol{y}+\boldsymbol{A} \frac{d \boldsymbol{y}}{d p}
$$

Multiplying $\boldsymbol{y}^{\dagger^{T}}$ to the both sides of this equation and integrating them on $0 \leq t \leq t_{k}$, we obtain

$$
\int_{0}^{t_{k}} d t\left\{\boldsymbol{y}^{\dagger^{T}} \frac{d}{d t}\left(\frac{d \boldsymbol{y}}{d p}\right)\right\}=\int_{0}^{t_{k}} d t\left(\boldsymbol{y}^{\dagger^{T}} \frac{d \boldsymbol{A}}{d p} \boldsymbol{y}\right)+\int_{0}^{t_{k}} d t\left(\boldsymbol{y}^{\dagger^{T}} \boldsymbol{A} \frac{d \boldsymbol{y}}{d p}\right)
$$

This equation can be rewritten as

$$
\left[\boldsymbol{y}^{\dagger^{T}} \frac{d \boldsymbol{y}}{d p}\right]_{0}^{t_{k}}=\int_{0}^{t_{k}} d t\left(\boldsymbol{y}^{\dagger^{T}} \frac{d \boldsymbol{A}}{d p} \boldsymbol{y}\right)+\int_{0}^{t_{k}} d t\left\{\frac{d \boldsymbol{y}^{T}}{d p}\left(\boldsymbol{A}^{T} \boldsymbol{y}^{\dagger}+\frac{d \boldsymbol{y}^{\dagger}}{d t}\right)\right\} .
$$

Note that

$$
\boldsymbol{y}^{\dagger^{T}} \boldsymbol{A} \frac{d \boldsymbol{y}}{d p}=\left(\boldsymbol{y}^{\dagger T} \boldsymbol{A} \frac{d \boldsymbol{y}}{d p}\right)^{T}=\frac{d \boldsymbol{y}^{T}}{d p} \boldsymbol{A}^{T} \boldsymbol{y}^{\dagger}
$$

since $\boldsymbol{y}^{\dagger^{T}} \boldsymbol{A} \frac{d \boldsymbol{y}}{d p}$ is scalar. Using Equation (10), Equation (17) is transformed to

$$
\boldsymbol{y}^{\dagger^{T}}\left(t_{k}\right) \frac{d \boldsymbol{y}\left(t_{k}\right)}{d p}-\boldsymbol{y}^{\dagger^{T}}(0) \frac{d \boldsymbol{y}(0)}{d p}=\int_{0}^{t_{k}} d t\left(\boldsymbol{y}^{\dagger^{T}} \frac{d \boldsymbol{A}}{d p} \boldsymbol{y}\right) .
$$

Since the initial condition for $\boldsymbol{y}$ is given, the second term of the left hand side of the above equation is zero. Now we are interested in a derivative of the number of neutrons 
to $p$ at $t=t_{k}$. In order to obtain this quantity, we set the final condition of $\boldsymbol{y}^{\dagger}$ as $\boldsymbol{y}^{\dagger}\left(t_{k}\right)=(1,0, \ldots, 0)^{T}$, and then we can obtain the following equation:

$$
\frac{\partial n\left(t_{k}\right)}{\partial p}=\int_{0}^{t_{k}} d t\left(\boldsymbol{y}^{\dagger^{T}} \frac{d \boldsymbol{A}}{d p} \boldsymbol{y}\right)
$$

Since the derivative $\frac{d \boldsymbol{A}}{d p}$ can be easily calculated, the derivative $\frac{\partial n}{\partial p}$ is also easily calculated using $\boldsymbol{y}$ and $\boldsymbol{y}^{\dagger}$.

The physical meaning of $\boldsymbol{y}^{\dagger}$ can be explained as follows. By multiplying $\boldsymbol{y}^{\dagger}$ to the both sides of Equation (9) and $\boldsymbol{y}^{T}$ to the both sides of Equation (10), integrating them over $t^{\prime} \leq t \leq t_{k}$, and subtracting one by another, we obtain the following equation:

$$
\left[\boldsymbol{y}^{T} \boldsymbol{y}^{\dagger}\right]_{t^{\prime}}^{t_{k}}=0
$$

Since $\boldsymbol{y}^{\dagger}\left(t_{k}\right)=(1,0, \ldots, 0)^{T}$, we obtain the following relation:

$$
n\left(t_{k}\right)=\boldsymbol{y}^{\dagger^{T}}\left(t^{\prime}\right) \boldsymbol{y}\left(t^{\prime}\right)=n^{\dagger}\left(t^{\prime}\right) n\left(t^{\prime}\right)+\sum_{i=1}^{N} C_{i}^{\dagger}\left(t^{\prime}\right) C_{i}\left(t^{\prime}\right)=\hat{n}\left(t^{\prime}\right)+\sum_{i=1}^{N} \hat{C}_{i}\left(t^{\prime}\right)
$$

From this equation, we can find that $\hat{n}\left(t^{\prime}\right)$ and $\hat{C}_{i}\left(t^{\prime}\right)$ are ancestors at $t=t^{\prime}$ of neutrons at $t=t_{k}$, and those divided by $n\left(t_{k}\right)$ are relative contributions of neutrons and fission product nuclides at $t=t^{\prime}$ to the neutrons at $t=t_{k}$.

While the averaged number of delayed neutrons emitted per a fission reaction $\nu_{d}$ is accurately measured generally, delayed neutron-related data such as independent fission yield and decay branching ratio for each fission product nuclide are not the case. Thus $\nu_{d}$ calculated from the delayed neutron data for fission product nuclides does not agree with $\nu_{d}$ given in a general-purpose nuclear data file. In order to reproduce $\nu_{d}$ given in the JENDL-4.0 nuclear data file from the microscopic nuclear data, we normalize all the fission yield values in the present study. This normalization should be considered when we calculate sensitivities to delayed neutron data which affect the $\nu_{d}$ value. For example, 
let us consider a sensitivity to a fission yield of nuclide $j, y_{j}$. When a perturbation $\Delta y_{j}$ is given to $y_{j}$, the following relation holds:

$$
\sum_{i=1}^{N}\left(y_{i}+\Delta y_{j} \delta_{i j}\right) D_{i}=\nu_{d}+\Delta y_{j} D_{j}
$$

where $\delta_{i j}$ is Kronecker's delta. This equation can be transformed to

$$
\sum_{i=1}^{N} \frac{\nu_{d}}{\nu_{d}+\Delta y_{j} D_{j}}\left(y_{i}+\Delta y_{j} \delta_{i j}\right) D_{i}=\sum_{i=1}^{N} y_{i}^{\prime} D_{i}=\nu_{d} .
$$

Thus a change in $y_{i}, \Delta y_{i}$, can be written as

$$
\Delta y_{i}=y_{i}^{\prime}-y_{i}=\frac{\Delta y_{j}}{\nu_{d}+\Delta y_{j} D_{j}}\left(\nu_{d} \delta_{i j}-D_{j} y_{i}\right) \approx \frac{\Delta y_{j}}{\nu_{d}}\left(\nu_{d} \delta_{i j}-D_{j} y_{i}\right)
$$

and the following equation is derived:

$$
\frac{d y_{i}}{d y_{j}}=\delta_{i j}-\frac{D_{j} y_{i}}{\nu_{d}}
$$

When the normalization of fission yields is considered, the constrained sensitivity of the reactor stable period $T$ to the fission yield $y_{j}, \bar{S}_{j}$, can be written as

$$
\begin{aligned}
\bar{S}_{j} & =\left(\frac{\partial T}{\partial y_{j}}\right)\left(\frac{y_{j}}{T}\right)=\sum_{i=1}^{N}\left(\frac{\partial T}{\partial y_{i}}\right)\left(\frac{y_{i}}{T}\right)\left(\frac{y_{j}}{y_{i}}\right)\left(\frac{d y_{i}}{d y_{j}}\right) \\
& =\sum_{i=1}^{N} S_{i} \frac{y_{j}}{y_{i}}\left(\delta_{i j}-\frac{D_{j} y_{i}}{\nu_{d}}\right)=S_{j}-\frac{D_{j} y_{j}}{\nu_{d}} \sum_{i=1}^{N} S_{i},
\end{aligned}
$$

where $S_{i}$ denotes the unconstrained sensitivity to $y_{i}$.

Note that another normalization is considered in the case of calculating sensitivities to a decay branching ratio; a sum of all the values of branching ratio should be unity. 


\section{Numerical result}

\subsection{Numerical procedure}

We numerically solve the one-point reactor kinetics equations (7) and (8) with a fixed value of $k_{p}$, and obtain a corresponding reactor stable period. Decay data of fission product nuclides such as decay constants, decay modes and their branching ratios are taken from the JENDL FP Decay Data File 2011 (JENDL/FPD-2011)[13]. Fission yield data are taken from the JENDL FP Fission Yields Data File 2011 (JENDL/FPY-2011)[13]. In the present calculation, thermal fissions by uranium-235 and plutonium-239, and fast fissions (at $500 \mathrm{keV}$ ) by plutonium-239 and uranium-238 are considered.

At an initial time step, a unit neutron and some delayed neutron precursors are introduced to a system. The number densities of the delayed neutron precursors at the initial time step, $C_{i}(0)$, is set as

$$
C_{i}(0)=\frac{k_{p} y_{i}}{\lambda_{i} l \nu_{p}}
$$

which is an estimate of the number densities in a critical state. The kinetics equation is solved with fine time steps $(\Delta t=0.1[\mathrm{~s}])$ under a constant (time-independent) reactivity: a fixed value of $k_{p}$. After a system reaches to its asymptotic state, a reactor stable period is calculated. Prompt neutron life time is assumed $1.5 \times 10^{-5}$ seconds for the thermal fission cases and $1.5 \times 10^{-7}$ seconds for the fast fission cases. The forward and adjoint kinetics equations are solved by the matrix exponential method with the Chebyshev rational approximation[14]. 


\subsection{Relation between reactivity and reactor stable period}

Relation between a reactivity and a reactor stable period is numerically obtained. In the present study, the prompt neutron multiplication factor is regarded as a reactivityrelated parameter for convenience. Figure 1 shows the relation between a prompt neutron multiplication factor and a reactor stable period above the delayed critical for thermal fissions as an example.

Next, we evaluate the decay chain effect on the relation between reactor stable period and prompt neutron multiplication factor for the thermal fission cases. Figure 2 shows a relative difference between two reactor stable periods deduced from the same prompt neutron multiplication factor; one is calculated with the decay chain effect consideration and the other is calculated without its consideration. When the chain effect is considered, delayed neutron precursors which are daughter nuclides of other fission product nuclides are produced by decay of the parent nuclides after a fission reaction, and then they emit delayed neutrons after decay. On the other hand, these delayed neutron precursors are generated instantaneously after a fission reaction and emit delayed neutrons according to their decay constants when the decay chain effect is neglected. Thus considering the decay chain effect makes the reactor stable period longer. The reason why the larger effect is observed in the uranium-235 fission will be discussed later.

\subsection{Ancestor quantifications}

As described in the preceding section, ancestors of neutrons at a certain time can be quantified with the function $\hat{C}_{i}(t)$ as shown in Equation (22). As an example, ancestors of neutrons are calculated for the uranium-235 thermal fission with different values of $k_{p}$ (or 
reactor stable period). Figures $\mathbf{3}$ to $\mathbf{5}$ show relative contributions of fission product nuclides iodine-137, bromine-88, -89, -90 and rubidium-94 as ancestors of neutrons at $t=0$. As the time $t$ approaches to zero, the contributions of fission products having long halflives such as iodine-137 (24.5 seconds) and bromine-88 (16.5 seconds) decrease and those having short half-lives such as rubidium-94 (2.7 seconds) and bromine-89 (4.4 seconds) increase. It is interesting to see that the contribution of each ancestor nuclide converges to a constant value as the time $t$ becomes far from zero. In order to see dependence of these converged values of ancestor contributions on a reactor stable period $T$, ancestor contributions are calculated at $t=-3 T$ with different values of $T$. Table 1 shows summary of the results. In the case of a long reactor stable period, fission product nuclides having long half-lives show large contributions. This result suggests that sensitivities of reactor stable period to nuclear data such as decay constants and fission yields depend on a value of a reactor stable period.

\subsection{Sensitivity calculations}

Sensitivities of reactor stable periods to fission yields and decay constants are calculated using Equations (14) and (20) with different values of reactor stable period. Note that all the sensitivities shown in the present section are relative ones: $\{(\partial T / \partial p) /(T / p)\}$.

Firstly two types of sensitivities, constrained and unconstrained sensitivities, are compared with each other. Figure 6 shows these two sensitivities to fission yields, which are calculated for the uranium-235 thermal fission with a reactor stable period of about 100 seconds. All the unconstrained sensitivities take negative values since an increase in the fission yield results in an increase of $\nu_{d}$, an averaged number of delayed neutrons. It is 
easily understood that the increase of $\nu_{d}$ under a constant value of $\nu_{p}$ makes the reactor stable period shorter since the averaged number of fission neutrons $\nu$ also increases. The magnitude of unconstrained sensitivities of nuclide $i$ is determined from an independent fission yield $y_{i}$, a total number of emitted neutrons per a nuclide generation $D_{i}$, and halflives of delayed neutron precursors which are generated from the nuclide $i$; the sensitivity of nuclide $i$ takes a large value if $y_{i}$ and $D_{i}$ are large and the half-lives of related precursors are short. Table 2 summarizes the fundamental data related to delayed neutron emission for some fission product nuclides. Since daughter nuclides of some of these nuclides such as germanium- 86 and selenium- 87 can be also delayed neutron precursors, an effective half-life is newly introduced here. A delayed neutron precursor $i$ with unit number density is introduced at an initial state and a time-dependent delayed neutron emission rate is numerically calculated. Then the effective half-life is defined as a time when a delayed neutron emission rate becomes half relative to its initial value. The effective half-life is also shown in Table 2. Please remember that time behavior of delayed neutron emission rate for these nuclides cannot be described by a unique exponential function. Taking selenium-87 for example, the delayed neutron emission rate increases for a while after the unit selenium-87 is introduced, and then it begins to decrease with the half-life of the daughter nuclide, bromine- 87 . The effective half-life of germanium-86 is longer than the physical half-life since its daughter nuclide arsenic-86 has long half-life of 0.945 second and large branching ratio to decay channel with neutron emission. The large value of the germanium-86 unconstrained sensitivity can be understood from a large value of $D_{i}$ and a small value of the effective half-life. On the other hand, even though the product of $y_{i}$ and $D_{i}$ of iodine-137 is also large, the iodine- 137 unconstrained sensitivity is not as large as the germanium- 86 sensitivity. This is because the half-life of iodine- 137 is much longer 
than the effective half-life of germanium- 86 .

The constrained sensitivities take both positive and negative values. Signs of constrained sensitivities are determined from (effective) half-lives of delayed neutron precursors. As shown in Equation (27), a difference between constrained and unconstrained sensitivities becomes significant if a product of $y_{i}$ and $D_{i}$ is large. When contribution of short-lived precursors such as germanium- 86 and bromine-90 becomes large, a reactor stable period becomes short. Thus the constrained sensitivities are negative. On the other hand, when contribution of long-lived precursors such as iodine-137 becomes large, a reactor stable period becomes long. In the following, only the constrained sensitivities will be considered.

Sensitivities of reactor stable periods to independent fission yields are shown in Tables 3 to 6 for various types of fissioning systems. Sensitivities whose absolute values are larger than 0.02 are only shown and relatively large sensitivities (larger than 0.1) are shown in bold characters. Sensitivities to fission yields of bromine isotopes in the uranium235 thermal fission are larger than those in other cases and those to fission yields of iodine isotopes in the plutonium-239 fission cases are larger than those in the uranium fission cases. A sensitivity to the germanium-86 fission yield is large only in the uranium-235 thermal fission case since a large fission yield value of 0.00628 is given in the uranium-235 thermal fission.

Similarly, sensitivity calculations are carried out without a consideration of the decay chain effect. In this case, sensitivities of reactor stable periods to not independent but cumulative fission yields are calculated. In the uranium-235 thermal fission case, a sensitivity to germanium-86 cumulative yield becomes very small and a sensitivity to arsenic-86 cumulative yield becomes large. These come from a fact that germanium-86 is not only 
a delayed neutron precursor but also a parent nuclide of different precursor arsenic-86 as described above. Branching ratios to decay channel with delayed neutron emissions of germanium-86 and arsenic-86 are 0.06 and 0.33 , respectively, and half-lives of germanium86 and arsenic- 86 are $87.8 \mathrm{~ms}$ and 0.945 s. Thus it can be said that germanium- 86 is rather important as a parent nuclide of a delayed neutron precursor arsenic-86.

Here let us consider the reason why the decay chain effect on reactor stable periods in the uranium-235 thermal fission case is larger than that in the plutonium-239 thermal fission case. Parent fission product nuclides of delayed neutron precursors, whose fission yields affect reactor stable periods, are germanium-86, selenium-87, antimony-137 and tellurium-137. As shown in Tables 3 and 4, sensitivities to fission yields of these nuclides in the uranium-235 thermal fission case are clearly larger than those in the plutonium-239 thermal fission case. Among of them, selenium-87 and tellurium-137 seem more important since those nuclides have relatively long half-lives: 5.29 seconds for selenium- 87 and 2.49 seconds for tellurium-137.

Finally, sensitivities of reactor stable periods to decay constants are shown from Tables 7 to 10. As a reactor stable period becomes shorter, sensitivities to decay constants of fission product nuclides having short half-lives become larger.

\subsection{Uncertainty quantifications}

Variances of independent fission yields, decay constants and decay branching ratios of fission product nuclides have been evaluated and given in JENDL/FPY-2011 and JENDL/FPD-2011. Using these variances and sensitivities calculated above, uncertainty of the reactor stable period can be quantified. Although there should be strong correla- 
tions among independent fission yields and among the fission yield and branching ratio of fission product nuclides having the same mass number, such correlations are ignored in the present study since the main purpose of the present study is to identify the important delayed neutron-related nuclear data for the reactor kinetics. Calculated uncertainties of reactor stable period are summarized in Table 11. In all the cases, dominant sources of uncertainties are fission yields and contributions of decay constant uncertainties are negligible. Total uncertainties of the plutonium-239 fast fission case are the largest and the those of the uranium-235 thermal case are the smallest. Notice again that these results are obtained without considering the correlations among fundamental delayed neutron data.

Figures 7 to 10 show nuclide-wise and nuclear data-wise uncertainties and Table 12 summarizes independent fission yields with their relative uncertainties of important fission product nuclides. In the uranium-235 thermal fission case, the fission yield uncertainty of germanium- 86 is a dominant contributor. This can be understood from a large sensitivity and a large uncertainty to/of independent fission yield of germanium-86 as shown in Tables 3 and 12. The fission yield of iodine-137, which has a large sensitivity, does not contribute to total uncertainty as much as the germanium- 86 since its uncertainty is very small in comparison with that of germanium-86. In the plutonium-239 thermal fission case, the fission yield uncertainties of selenium-87, yttrium-98m and iodine-137 are dominant contributors. The branching ratio uncertainty of yttrium-98m is also a dominant contributor in this case. In the plutonium-239 fast fission case, the fission yield uncertainty of iodine-137 is about twice as those in the thermal fission case. This comes from a difference in uncertainties of iodine-137 independent fission yield as shown in Table 12 . Contributions of bromine-88, rubidium-94 and -95 independent fission yield uncertainties are also large because of the large values of independent yield uncertainties. In the 
uranium-238 fast fission case, the fission yield uncertainties of bromine-91, tellurium-137 and iodine-137, some of which do not contribute to the uncertainty at all in other cases, are dominant contributors.

\section{Conclusion}

In order to better predict kinetic behavior of a nuclear fission reactor, improvement of delayed neutron parameters is essential. Such improvement can be realized by efforts done in the nuclear physics field, and it is required to establish a path from the microscopic nuclear data to the macroscopic delayed neutron parameters. The present paper has identified important nuclear data for reactor kinetics with a help of the adjoint kinetics equation and it has been found that they are dependent on an inserted reactivity (or a reactor stable period). Furthermore, nuclide-wise and nuclear data-wise uncertainties of the reactor stable period have been quantified using the variance data given in the nuclear data file, and the nuclear data which require further improvement have been identified.

The authors believe that the results obtained through the present study are helpful for the nuclear data community to improve delayed neutron-related nuclear data. We hope that more accurate and reliable delayed neutron data will be realized by joint contributions of the microscopic and macroscopic approaches in future.

\section{Acknowledgment}

The authors greatly appreciate reviewers' helpful comments and suggestions on this manuscript. This work was supported by JSPS KAKENHI Grant Number 24561040. 


\section{References}

1) Keepin GR, Wimett TF, Ziegler's RK. Delayed neutrons from fissionable isotopes of uranium, plutonium, and thorium. Physical Review. 1957;107;1044-1049.

2) Tuttle RJ. Delayed-neutron data for reactor-physics analysis. Nucl. Sci. Eng. 1975;56;37-71.

3) Spriggs GD, Campbell JM. An 8-group delayed neutron model based on a consistent set of half-lives. Prog. Nucl. Energy. 2002;41;223-251.

4) Brady MC, England TR. Delayed neutron data and group parameters for 43 fissioning systems. Nucl. Sci. Eng. 1989;103;129-149.

5) Spriggs GD. In-pile measurement of the decay constants and relative abundances of delayed neutrons. Nucl. Sci. Eng. 1993;114;342-351.

6) Williams T. On the choice of delayed neutron parameters for the analysis of kinetics experiments in ${ }^{235}$ U systems. Ann. Nucl. Energy. 1996;23;1261-1265.

7) Oyamatsu K. Reactor kinetics calculated in the summation method. J. Nucl. Sci. Technol. Supp. 2. $2002 ; 1109-1111$.

8) Chiba G, Tsuji M, Narabayashi T. Sensitivity analysis for reactor period induced by positive reactivity using one-point adjoint kinetics equation. Paper presented at: Int. Conf. on Nuclear Data for Science and Technology, ND2013; 2013 March 4-8; New York, NY.

9) Lewins J. The time-dependent importance of neutrons and precursors. Nucl. Sci. Eng. 1960;7;268-274.

10) van Rooijen WFG., Lathouwers D. Sensitivity analysis for delayed neutron data. Ann. Nucl. Energy. $2008 ; 35 ; 2186-2194$.

11) Onega RJ. A reformulation of the reactor kinetics equations. Nucl. Sci. Eng. 1971;43;345-348.

12) Miyazono T, Sagisaka M, Ohta H, Oyamatsu K, Tamaki M. Delayed neutron spectra and their uncertainties in fission product summation calculations. In: Iguchi T, Fukahori T. Proc. of the 1996 
J. Nucl. Sci. \& Technol.

Symposium on Nuclear Data;1996 Nov. 21-22; Ibaraki (Japan): JAERI-Conf 97-005: Japan Atomic Energy Research Institute; 1997;83-88.

13) Katakura J. JENDL FP decay data file 2011 and fission yields data file 2011. JAEA-Data/Code 2011-025. Ibaraki (Japan): Japan Atomic Energy Agency; 2011.

14) Pusa M, Leppänen J. Computing the matrix exponential in burnup calculations. Nucl. Sci. Eng. 2010;164;140-150. 
Table 1 Ancestor contributions dependent on reactor stable period

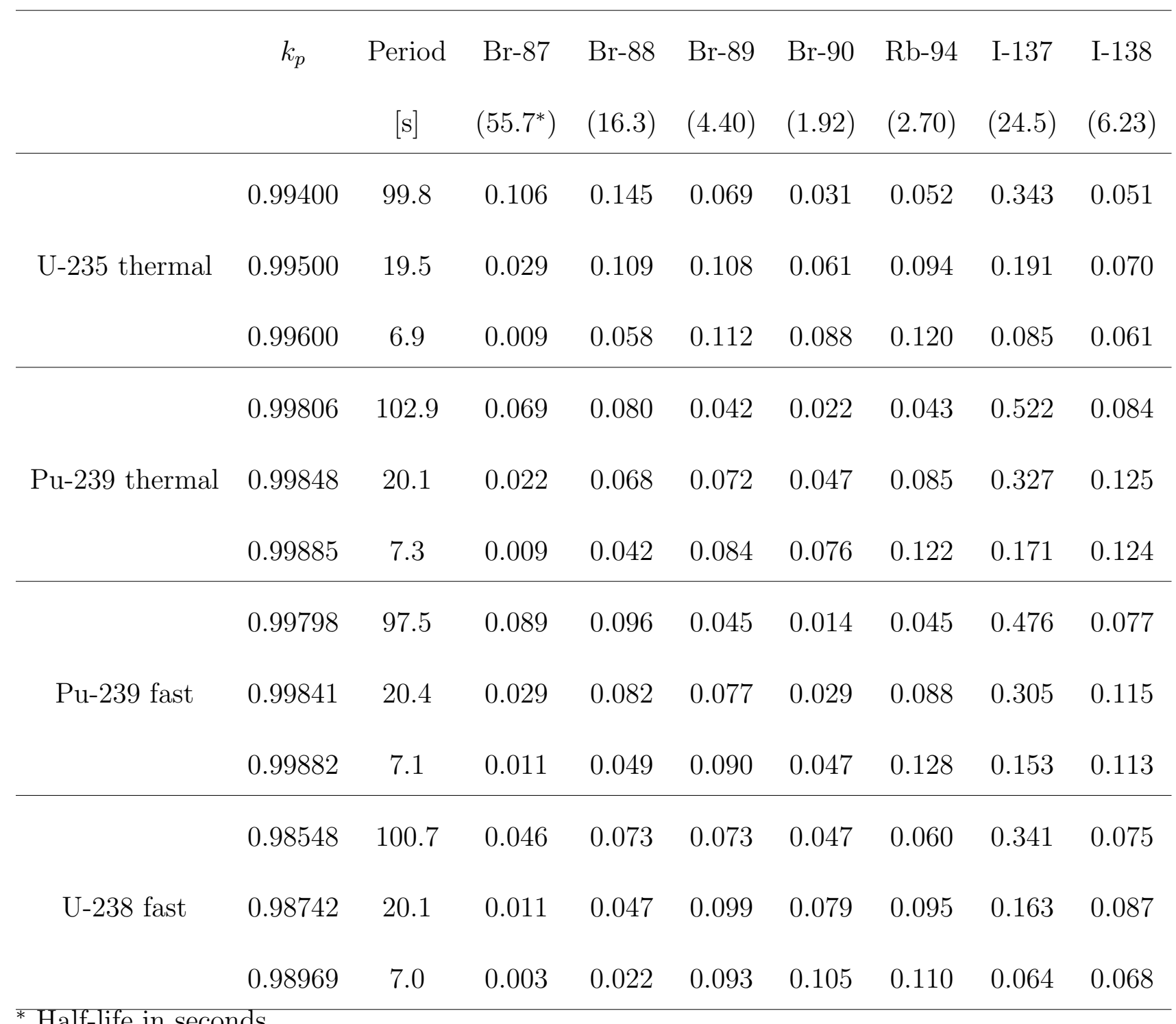


Table 2 Fundamental data related to delayed neutron emission

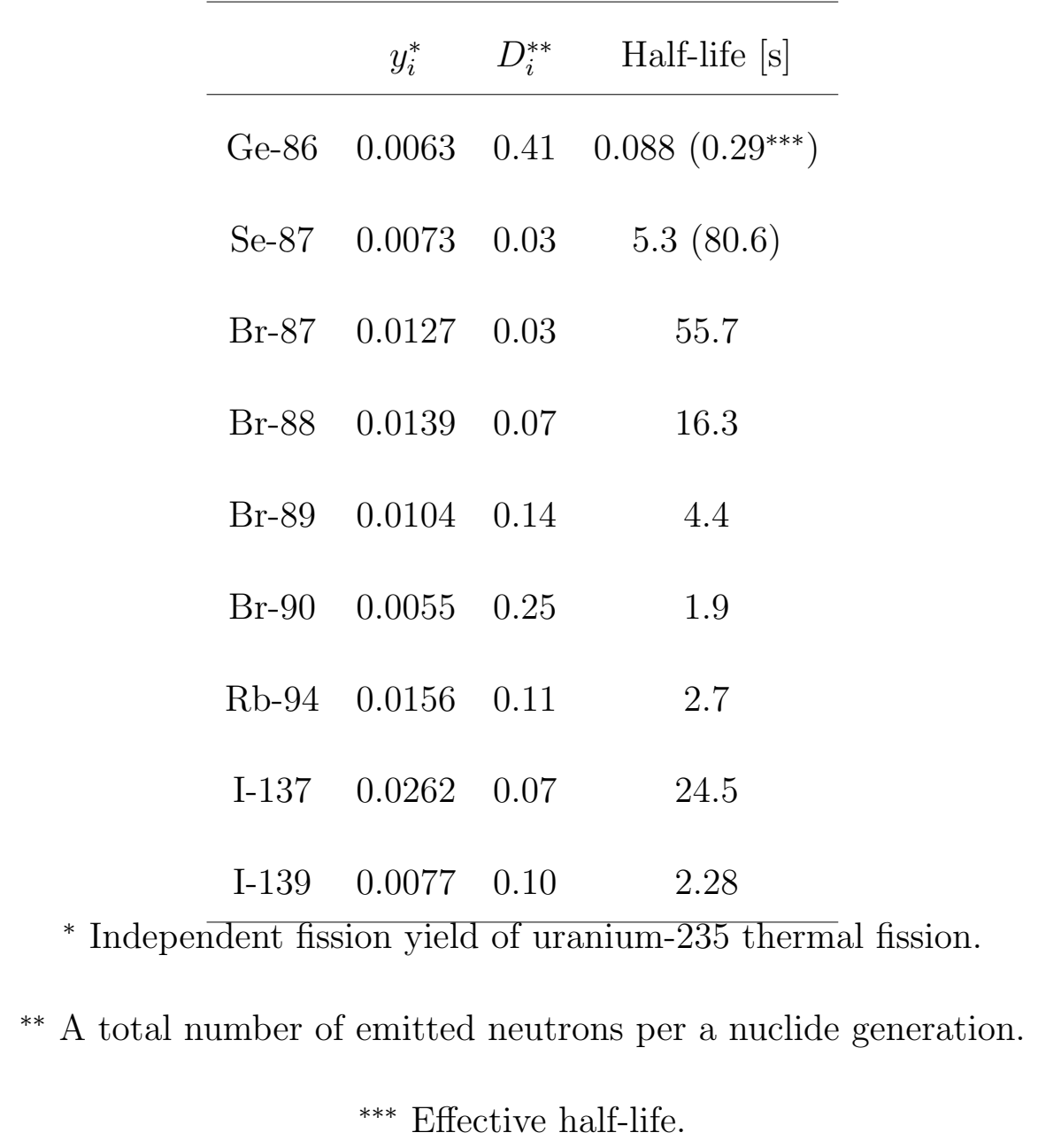


Table 3 Sensitivities of reactor stable period to fission yield of uranium-235 thermal fission

\begin{tabular}{|c|c|c|c|}
\hline & \multicolumn{3}{|c|}{ Reactor stable period $[\mathrm{s}]$} \\
\hline & 99.8 & 19.5 & 6.9 \\
\hline Ge-86 & -0.134 & -0.165 & -0.169 \\
\hline As- 85 & -0.029 & -0.029 & -0.019 \\
\hline Se- 87 & 0.064 & 0.047 & 0.053 \\
\hline $\mathrm{Br}-87$ & 0.101 & 0.074 & 0.053 \\
\hline $\mathrm{Br}-88$ & 0.084 & 0.112 & 0.106 \\
\hline $\mathrm{Br}-89$ & -0.022 & 0.007 & 0.040 \\
\hline $\mathrm{Br}-90$ & -0.059 & -0.059 & -0.042 \\
\hline $\mathrm{Br}-91$ & -0.026 & -0.034 & -0.038 \\
\hline Rb-94 & -0.055 & -0.042 & -0.013 \\
\hline Rb-95 & -0.040 & -0.053 & -0.063 \\
\hline Rb-96 & -0.014 & -0.019 & -0.023 \\
\hline Y-98m & -0.026 & -0.026 & -0.018 \\
\hline Sb-137 & -0.018 & -0.026 & -0.034 \\
\hline Te-136 & 0.018 & 0.023 & 0.021 \\
\hline Te-137 & 0.042 & 0.046 & 0.040 \\
\hline $\mathrm{I}-137$ & 0.282 & 0.302 & 0.254 \\
\hline I-139 & -0.030 & -0.027 & 0.043 \\
\hline
\end{tabular}


Table 4 Sensitivities of reactor stable period to fission yield of plutonium-239 thermal fission

\begin{tabular}{|c|c|c|c|}
\hline & \multicolumn{3}{|c|}{ Reactor stable period $[\mathrm{s}]$} \\
\hline & 102.9 & 19.5 & 6.9 \\
\hline Se-87 & 0.021 & 0.016 & 0.012 \\
\hline $\mathrm{Br}-87$ & 0.077 & 0.060 & 0.045 \\
\hline $\mathrm{Br}-88$ & 0.041 & 0.061 & 0.064 \\
\hline Br-89 & -0.033 & -0.022 & -0.000 \\
\hline Br-90 & -0.065 & -0.078 & -0.072 \\
\hline Rb-94 & -0.077 & -0.082 & -0.061 \\
\hline Rb-95 & -0.058 & -0.084 & -0.107 \\
\hline Y-98m & -0.082 & -0.097 & -0.088 \\
\hline Y-99 & -0.036 & -0.045 & -0.047 \\
\hline Sb-135 & -0.018 & -0.023 & -0.022 \\
\hline Te-137 & 0.021 & 0.025 & 0.022 \\
\hline $\mathrm{I}-137$ & 0.399 & 0.461 & 0.415 \\
\hline $\mathrm{I}-139$ & -0.037 & -0.042 & -0.036 \\
\hline
\end{tabular}


Table 5 Sensitivities of reactor stable period to fission yield of plutonium-239 fast fission

\begin{tabular}{|c|c|c|c|}
\hline & \multicolumn{3}{|c|}{ Reactor stable period $[\mathrm{s}]$} \\
\hline & 97.5 & 20.4 & 7.1 \\
\hline As- 85 & -0.034 & -0.040 & -0.037 \\
\hline Se- 87 & 0.031 & 0.024 & 0.018 \\
\hline $\mathrm{Br}-87$ & 0.096 & 0.075 & 0.057 \\
\hline $\mathrm{Br}-88$ & 0.047 & 0.070 & 0.074 \\
\hline $\mathrm{Br}-89$ & -0.036 & -0.025 & -0.002 \\
\hline Br-90 & -0.041 & -0.048 & -0.046 \\
\hline Rb-94 & -0.082 & -0.088 & -0.067 \\
\hline Rb-95 & -0.045 & -0.064 & -0.082 \\
\hline Rb-96 & -0.015 & -0.021 & -0.028 \\
\hline Y-98m & -0.085 & -0.100 & -0.093 \\
\hline Y-99 & -0.036 & -0.046 & -0.048 \\
\hline $\mathrm{Y}-100 \mathrm{~m}$ & -0.018 & -0.024 & -0.028 \\
\hline $\mathrm{Sb}-135$ & -0.017 & -0.021 & -0.021 \\
\hline Te-136 & 0.015 & 0.021 & 0.021 \\
\hline Te-137 & 0.021 & 0.024 & 0.022 \\
\hline $\mathrm{I}-137$ & 0.362 & 0.419 & 0.380 \\
\hline $\mathrm{I}-138$ & -0.025 & 0.004 & 0.037 \\
\hline I-139 & -0.026 & -0.030 & -0.026 \\
\hline
\end{tabular}


Table 6 Sensitivities of reactor stable period to fission yield of uranium-238 fast fission

\begin{tabular}{|c|c|c|c|}
\hline & \multicolumn{3}{|c|}{ Reactor stable period $[\mathrm{s}$} \\
\hline & 100.7 & 20.1 & 7.0 \\
\hline As-85 & -0.033 & -0.027 & -0.012 \\
\hline Se-87 & 0.046 & 0.030 & 0.020 \\
\hline Se-88 & 0.025 & 0.028 & 0.024 \\
\hline $\mathrm{Br}-87$ & 0.032 & 0.021 & 0.014 \\
\hline $\mathrm{Br}-88$ & 0.038 & 0.043 & 0.037 \\
\hline Br-89 & 0.015 & 0.025 & 0.045 \\
\hline Br-90 & -0.051 & -0.044 & -0.024 \\
\hline Br-91 & -0.037 & -0.046 & -0.050 \\
\hline Br-92 & -0.020 & -0.012 & -0.029 \\
\hline Rb-94 & -0.027 & -0.012 & 0.009 \\
\hline Rb-95 & -0.036 & -0.045 & -0.051 \\
\hline Rb-96 & -0.042 & -0.054 & -0.065 \\
\hline Sb-135 & -0.038 & -0.036 & -0.024 \\
\hline Te-136 & 0.033 & 0.035 & 0.029 \\
\hline Te-137 & 0.116 & 0.109 & 0.086 \\
\hline $\mathrm{I}-137$ & 0.250 & 0.233 & 0.178 \\
\hline $\mathrm{I}-138$ & 0.020 & 0.044 & 0.056 \\
\hline I-139 & -0.027 & -0.019 & -0.004 \\
\hline $\mathrm{I}-140$ & -0.018 & -0.021 & -0.021 \\
\hline Cs-145 & -0.020 & -0.024 & -0.026 \\
\hline
\end{tabular}


Table 7 Sensitivities of reactor stable period to decay constant in uranium-235 thermal fission

\begin{tabular}{|c|c|c|c|}
\hline & \multicolumn{3}{|c|}{ Reactor stable period $[\mathrm{s}]$} \\
\hline & 99.8 & 19.5 & 6.9 \\
\hline As-85 $\left(2.03^{*}\right)$ & -0.022 & -0.042 & -0.060 \\
\hline As-86 (0.95) & -0.022 & -0.048 & -0.082 \\
\hline $\mathrm{Br}-87(55.7)$ & -0.107 & -0.030 & -0.009 \\
\hline $\mathrm{Br}-88(16.3)$ & -0.145 & -0.109 & -0.057 \\
\hline $\operatorname{Br}-89(4.40)$ & -0.069 & -0.108 & -0.112 \\
\hline Br-90 (1.92) & -0.031 & -0.061 & -0.087 \\
\hline Rb-93 (5.84) & -0.028 & -0.040 & -0.036 \\
\hline Rb-94 (2.70) & -0.052 & -0.094 & -0.120 \\
\hline Y-98m (2.0) & -0.014 & -0.028 & -0.040 \\
\hline Y-99 (1.48) & -0.007 & -0.014 & -0.021 \\
\hline Te-136 (17.6) & -0.024 & -0.017 & -0.009 \\
\hline I-137 (24.5) & -0.343 & -0.191 & -0.085 \\
\hline $\mathrm{I}-138(6.23)$ & -0.051 & -0.070 & -0.061 \\
\hline I-139 (2.28) & -0.020 & -0.039 & -0.053 \\
\hline
\end{tabular}


Table 8 Sensitivities of reactor stable period to decay constant in plutonium-239 thermal fission

\begin{tabular}{cccc}
\hline & \multicolumn{3}{c}{ Reactor stable period $[\mathrm{s}]$} \\
& 102.9 & 19.5 & 6.9 \\
\hline $\operatorname{Br}-87(55.7)$ & -0.070 & -0.022 & -0.009 \\
Br-88 (16.3) & -0.080 & -0.068 & -0.042 \\
Br-89 (4.40) & -0.042 & -0.072 & -0.084 \\
Br-90 (1.92) & -0.022 & -0.047 & -0.076 \\
\hline Rb-93 (5.84) & -0.022 & -0.034 & -0.034 \\
Rb-94 (2.70) & -0.043 & -0.086 & $\mathbf{- 0 . 1 2 2}$ \\
\hline Y-98m (2.0) & -0.029 & -0.062 & -0.098 \\
\hline Y-99 (1.48) & -0.009 & -0.020 & $\mathbf{- 0 . 0 3 5}$ \\
\hline I-137 (24.5) & $\mathbf{- 0 . 5 2 2}$ & $\mathbf{- 0 . 3 2 7}$ & $\mathbf{- 0 . 1 6 4}$ \\
I-138 (6.23) & -0.084 & $\mathbf{- 0 . 1 2 5}$ & $\mathbf{- 0 . 1 2 3}$ \\
\hline I-139 (2.28) & -0.016 & $\mathbf{- 0 . 0 3 3}$ & $\mathbf{- 0 . 0 5 0}$ \\
\hline
\end{tabular}


Table 9 Sensitivities of reactor stable period to decay constant in plutonium-239 fast fission

\begin{tabular}{|c|c|c|c|}
\hline & \multicolumn{3}{|c|}{ Reactor stable period $[\mathrm{s}]$} \\
\hline & 97.5 & 20.4 & 7.1 \\
\hline As-85 (2.03) & -0.012 & -0.026 & -0.041 \\
\hline $\operatorname{Br}-87(55.7)$ & -0.042 & -0.029 & -0.011 \\
\hline $\operatorname{Br}-88(16.3)$ & -0.102 & -0.082 & -0.049 \\
\hline $\operatorname{Br}-89(4.40)$ & -0.046 & -0.077 & -0.091 \\
\hline Br-90 (1.92) & -0.014 & -0.029 & -0.047 \\
\hline Rb-93 (5.84) & -0.029 & -0.042 & -0.043 \\
\hline Rb-94 (2.70) & -0.046 & -0.088 & -0.129 \\
\hline Y-98m (2.0) & -0.030 & -0.062 & -0.101 \\
\hline Y-99 (1.48) & -0.009 & -0.020 & -0.035 \\
\hline Te-136 (17.6) & -0.026 & -0.020 & -0.012 \\
\hline $\mathrm{I}-137(24.5)$ & -0.505 & -0.305 & -0.155 \\
\hline $\mathrm{I}-138(6.23)$ & -0.079 & -0.115 & -0.114 \\
\hline $\mathrm{I}-139(2.28)$ & -0.011 & -0.022 & -0.035 \\
\hline
\end{tabular}


Table 10 Sensitivities of reactor stable period to decay constant in uranium-238 fast fission

\begin{tabular}{|c|c|c|c|}
\hline & \multicolumn{3}{|c|}{ Reactor stable period $[\mathrm{s}]$} \\
\hline & 100.7 & 20.1 & 7.0 \\
\hline As-85 (2.03) & -0.032 & -0.054 & -0.070 \\
\hline $\mathrm{Br}-87(55.7)$ & -0.056 & -0.011 & -0.003 \\
\hline $\mathrm{Br}-88(16.3)$ & -0.072 & -0.047 & -0.022 \\
\hline $\operatorname{Br}-89(4.40)$ & -0.073 & -0.099 & -0.093 \\
\hline Br-90 (1.92) & -0.046 & -0.079 & -0.105 \\
\hline Rb-93 (5.84) & -0.019 & -0.023 & -0.018 \\
\hline Rb-94 (2.70) & -0.060 & -0.095 & -0.110 \\
\hline Y-98m (2.0) & -0.012 & -0.020 & -0.026 \\
\hline Y-99 (1.48) & -0.008 & -0.016 & -0.023 \\
\hline Te-136 (17.6) & -0.038 & -0.025 & -0.012 \\
\hline Te-137 (2.49) & -0.021 & -0.023 & -0.021 \\
\hline I-137 (24.5) & -0.336 & -0.164 & -0.064 \\
\hline $\mathrm{I}-138(6.23)$ & -0.074 & -0.087 & -0.068 \\
\hline I-139 (2.28) & -0.033 & -0.055 & -0.069 \\
\hline
\end{tabular}


Table 11 reactor stable period uncertainties

\begin{tabular}{|c|c|c|c|c|c|}
\hline & \multirow{2}{*}{$\begin{array}{c}\text { Period } \\
\qquad[\mathrm{s}]\end{array}$} & \multicolumn{4}{|c|}{ Relative uncertainty [\%] } \\
\hline & & Total & Decay constant & Fission yield & Branching ratio \\
\hline \multirow{3}{*}{ U-235 thermal } & 99.8 & 4.4 & 0.3 & 3.7 & 2.4 \\
\hline & 19.5 & 5.0 & 0.4 & 4.4 & 2.5 \\
\hline & 6.9 & 5.0 & 0.5 & 4.5 & 2.1 \\
\hline \multirow{3}{*}{$\mathrm{Pu}-239$ thermal } & 102.9 & 6.0 & 0.5 & 5.1 & 3.0 \\
\hline & 19.5 & 7.1 & 0.7 & 6.1 & 3.5 \\
\hline & 6.9 & 6.9 & 1.0 & 6.0 & 3.2 \\
\hline \multirow{3}{*}{$\mathrm{Pu}-239$ fast } & 97.5 & 11.7 & 0.5 & 11.2 & 3.3 \\
\hline & 20.4 & 13.6 & 0.7 & 13.0 & 3.8 \\
\hline & 7.1 & 13.0 & 1.0 & 12.5 & 3.6 \\
\hline \multirow{3}{*}{$\mathrm{U}-238$ fast } & 100.7 & 8.6 & 0.4 & 8.3 & 2.1 \\
\hline & 20.1 & 8.8 & 0.4 & 8.5 & 1.9 \\
\hline & 7.0 & 8.1 & 0.5 & 7.9 & 1.5 \\
\hline
\end{tabular}


Table 12 Independent fission yields and their uncertainties given in JENDL/FPY-2011

\begin{tabular}{ccccc}
\hline & U-235 thermal & Pu-239 thermal & Pu-239 fast & U-238 fast \\
\hline Ge-86 & $0.00628\left(16^{*}\right)$ & $<0.00001(64)$ & $<0.00001(64)$ & $0.00006(64)$ \\
Se-87 & $0.00730(4)$ & $0.00137(64)$ & $0.00180(64)$ & $0.00849(32)$ \\
Br-88 & $0.01386(2)$ & $0.00465(4)$ & $0.00486(64)$ & $0.00901(45)$ \\
Br-91 & $0.00223(11)$ & $0.00020(64)$ & $0.00033(64)$ & $0.00760(64)$ \\
Rb-94 & $0.01564(2.8)$ & $0.00704(16)$ & $0.00648(64)$ & $0.02540(32)$ \\
Rb-95 & $0.00763(4)$ & $0.00432(23)$ & $0.00289(64)$ & $0.01598(32)$ \\
Rb-96 & $0.00038(8)$ & $0.00039(64)$ & $0.00060(64)$ & $0.01171(32)$ \\
Y-98m & $0.01874(32)$ & $0.02013(32)$ & $0.01808(32)$ & $0.02622(32)$ \\
Sb-137 & $0.00074(64)$ & $0.00000(64)$ & $0.00000(64)$ & $0.00038(64)$ \\
Te-137 & $0.00392(8)$ & $0.00129(64)$ & $0.00115(64)$ & $0.01570(32)$ \\
I-137 & $0.02619(4)$ & $0.02299(6)$ & $0.01888(16)$ & $0.03537(16)$ \\
\hline & & Uncertainty in percent. & \\
\hline
\end{tabular}




\section{Figure captions}

Figure 1. Relation between prompt neutron multiplication factor and reactor stable period for thermal fission

Figure 2. Decay chain effect on reactor stable period corresponding prompt neutron multiplication factor for thermal fission. The Decay chain effect is defined as a relative difference between two reactor stable periods; one is calculated with the decay chain effect consideration and the other is calculated without its consideration.

Figure 3. Relative contributions of fission product nuclides as ancestors of neutrons at $t=0$ in uranium-235 thermal fission $(T=100[\mathrm{sec}])$

Figure 4. Relative contributions of fission product nuclides as ancestors of neutrons at $t=0$ in uranium-235 thermal fission $(T=20[\mathrm{sec}])$

Figure 5. Relative contributions of fission product nuclides as ancestors of neutrons at $t=0$ in uranium-235 thermal fission $(T=7[\mathrm{sec}])$

Figure 6. Constrained and unconstrained sensitivities of reactor stable period to fission yield for uranium-235 thermal fission $(T=100[\mathrm{sec}])$

Figure 7. Nuclide-wise and Nuclear data-wise uncertainties of reactor stable period in the uranium-235 thermal fission case

Figure 8. Nuclide-wise and Nuclear data-wise uncertainties of reactor stable 
period in the plutonium-239 thermal fission case

Figure 9. Nuclide-wise and Nuclear data-wise uncertainties of reactor stable period in the plutonium-239 fast fission case

Figure 10. Nuclide-wise and Nuclear data-wise uncertainties of reactor stable period in the uranium-238 fast fission case 


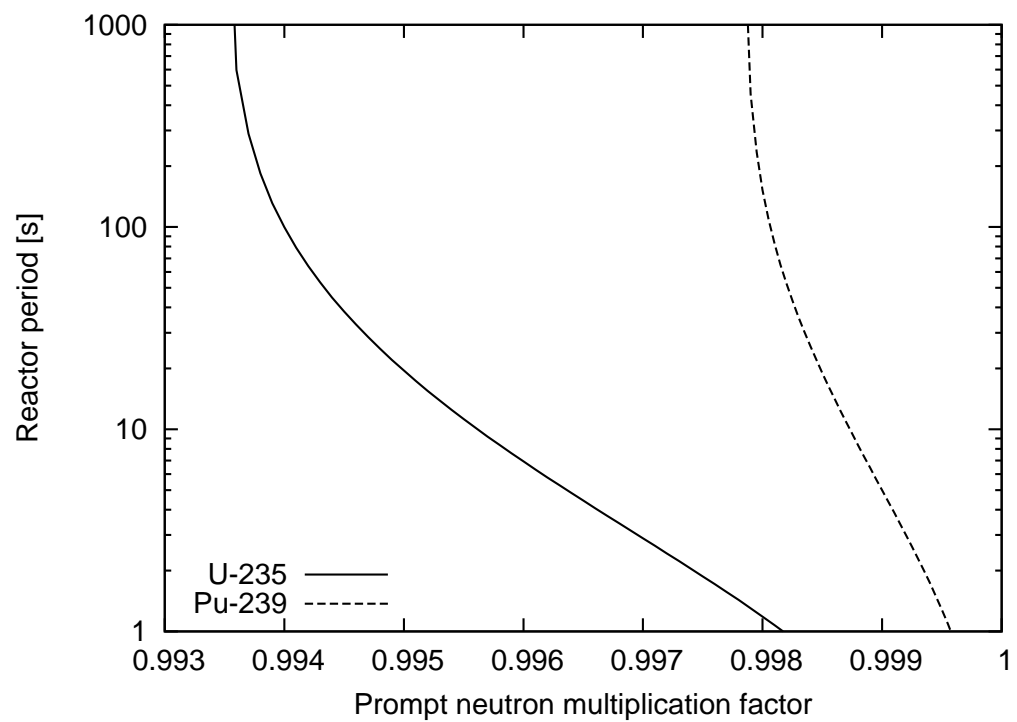

Figure 1 Relation between prompt neutron multiplication factor and reactor stable period for thermal fission

\section{G.Chiba:}

Sensitivity and uncertainty analysis for reactor stable period induced by positive reactivity using one-point adjoint kinetics equation 


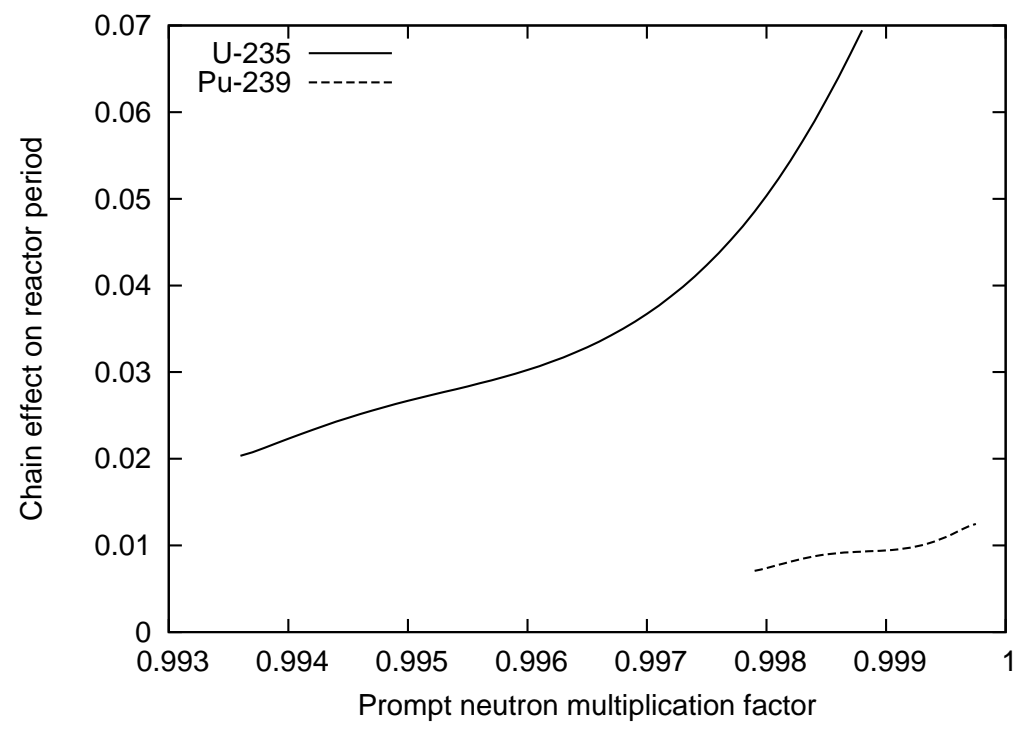

Figure 2 Decay chain effect on reactor stable period corresponding prompt neutron multiplication factor for thermal fission. The Decay chain effect is defined as a relative difference between two reactor stable periods; one is calculated with the decay chain effect consideration and the other is calculated without its consideration.

\section{G.Chiba:}

Sensitivity and uncertainty analysis for reactor stable period induced by positive reactivity using one-point adjoint kinetics equation 


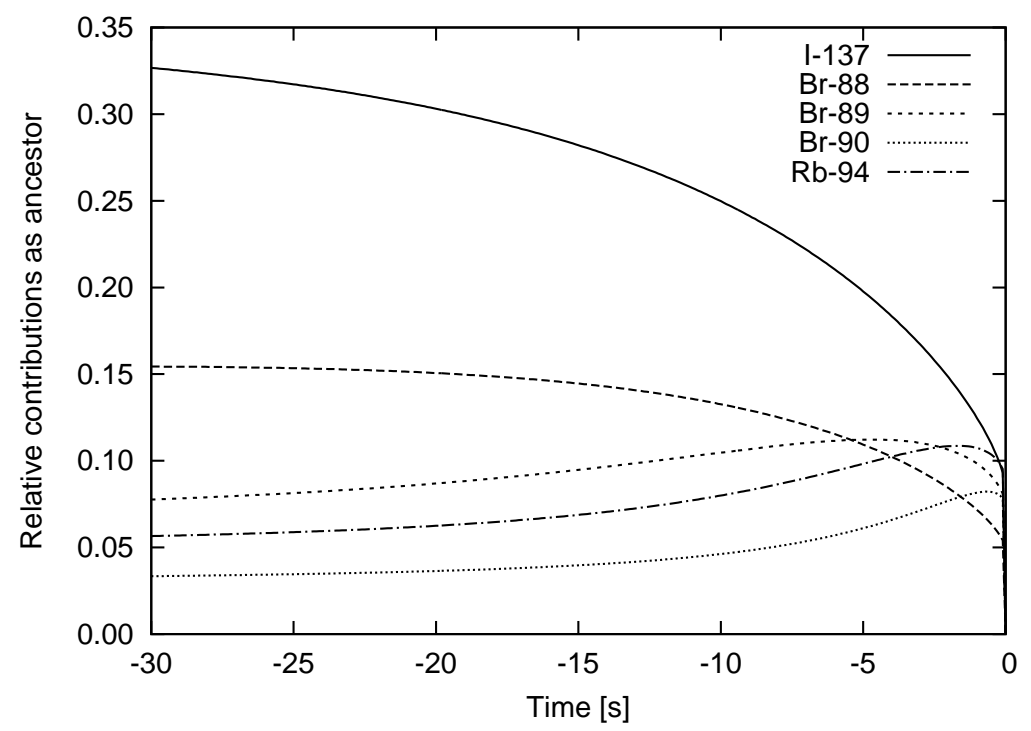

Figure 3 Relative contributions of fission product nuclides as ancestors of neutrons at $t=0$ in uranium235 thermal fission $(T=100[\mathrm{sec}])$

\section{G.Chiba:}

Sensitivity and uncertainty analysis for reactor stable period induced by positive reactivity using one-point adjoint kinetics equation 


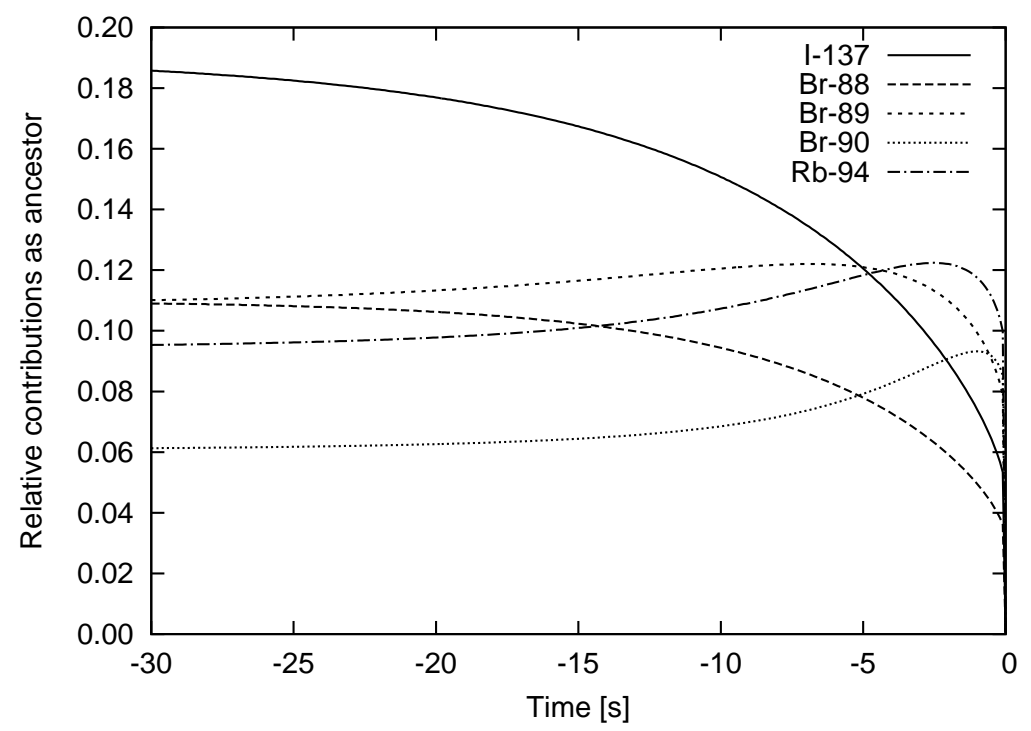

Figure 4 Relative contributions of fission product nuclides as ancestors of neutrons at $t=0$ in uranium235 thermal fission $(T=20[\mathrm{sec}])$

\section{G.Chiba:}

Sensitivity and uncertainty analysis for reactor stable period induced by positive reactivity using one-point adjoint kinetics equation 


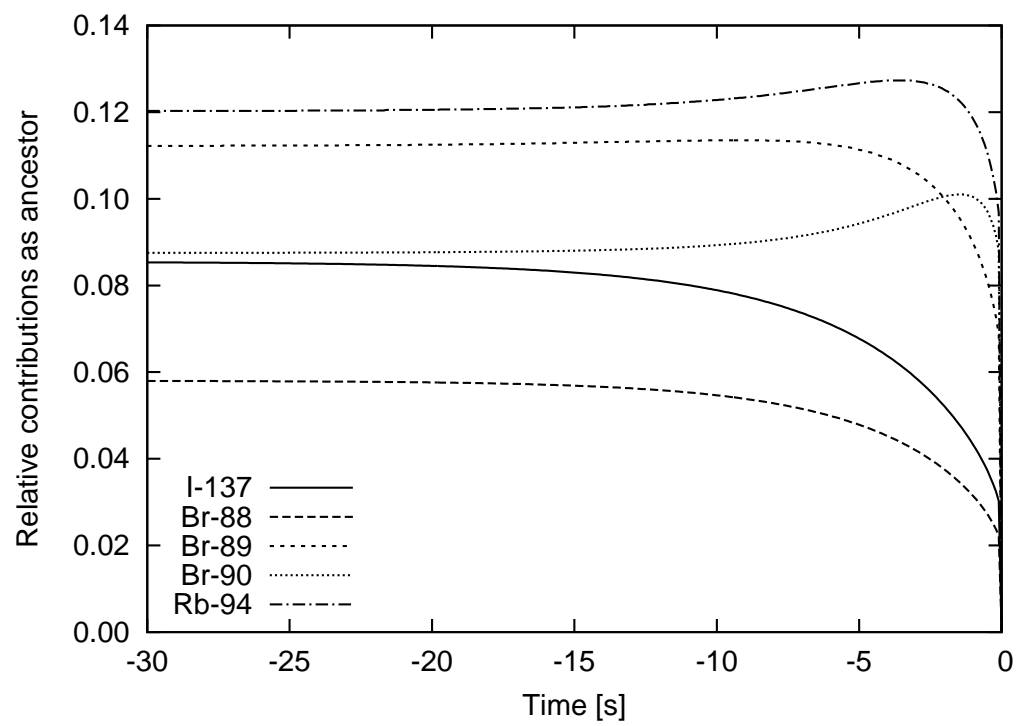

Figure 5 Relative contributions of fission product nuclides as ancestors of neutrons at $t=0$ in uranium235 thermal fission $(T=7[\mathrm{sec}])$

\section{G.Chiba:}

Sensitivity and uncertainty analysis for reactor stable period induced by positive reactivity using one-point adjoint kinetics equation 


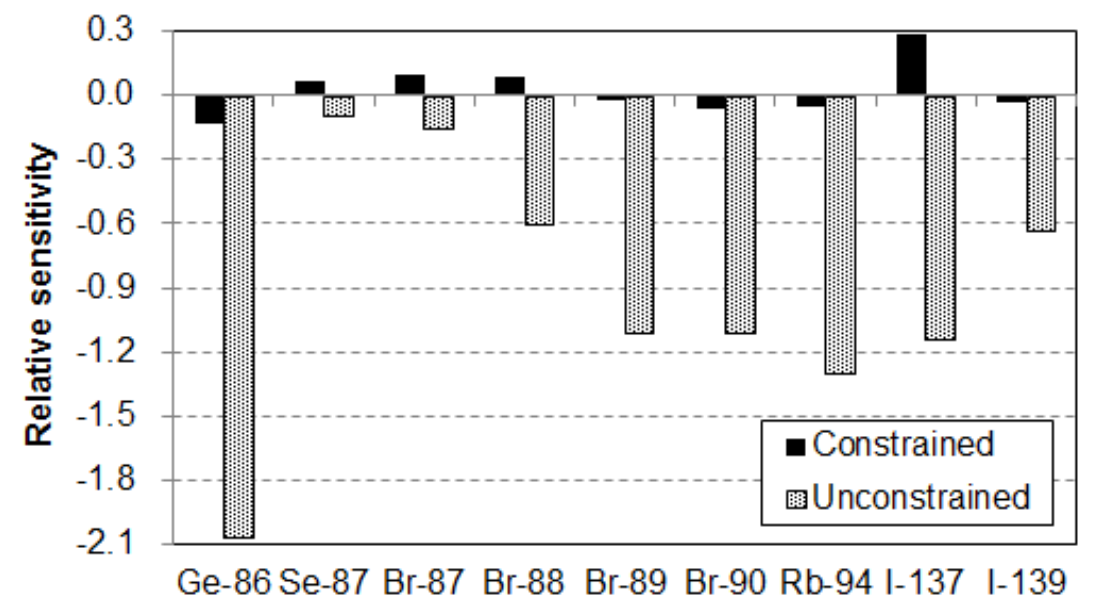

Figure 6 Constrained and unconstrained sensitivities of reactor stable period to fission yield for uranium-235 thermal fission $(T=100[\mathrm{sec}])$

\section{G.Chiba:}

Sensitivity and uncertainty analysis for reactor stable period induced by positive reactivity using one-point adjoint kinetics equation 


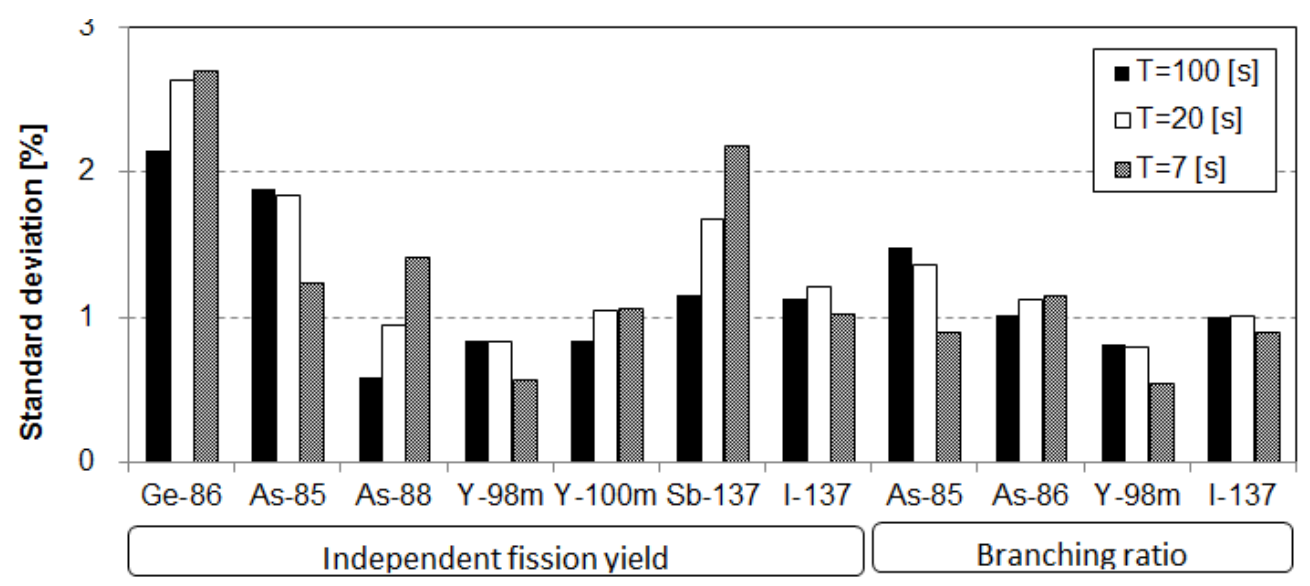

Figure 7 Nuclide-wise and Nuclear data-wise uncertainties of reactor stable period in the uranium-235 thermal fission case

G.Chiba:

Sensitivity and uncertainty analysis for reactor stable period induced by positive reactivity using one-point adjoint kinetics equation 


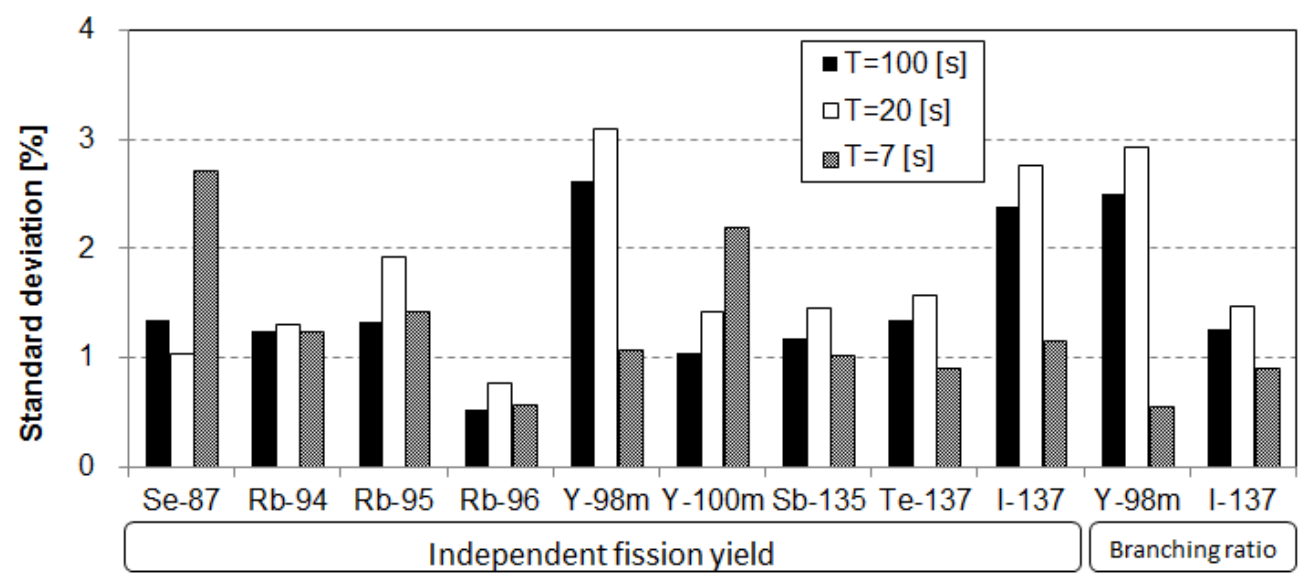

Figure 8 Nuclide-wise and Nuclear data-wise uncertainties of reactor stable period in the plutonium-239 thermal fission case

\section{G.Chiba:}

Sensitivity and uncertainty analysis for reactor stable period induced by positive reactivity using one-point adjoint kinetics equation 


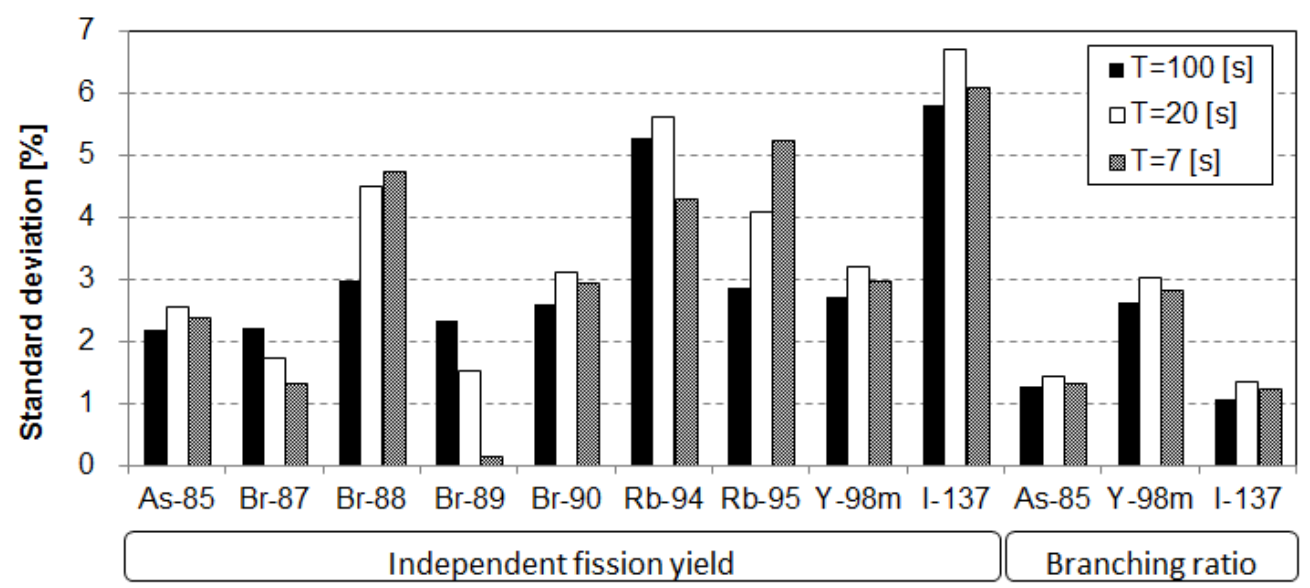

Figure 9 Nuclide-wise and Nuclear data-wise uncertainties of reactor stable period in the plutonium-239 fast fission case

\section{G.Chiba:}

Sensitivity and uncertainty analysis for reactor stable period induced by positive reactivity using one-point adjoint kinetics equation 


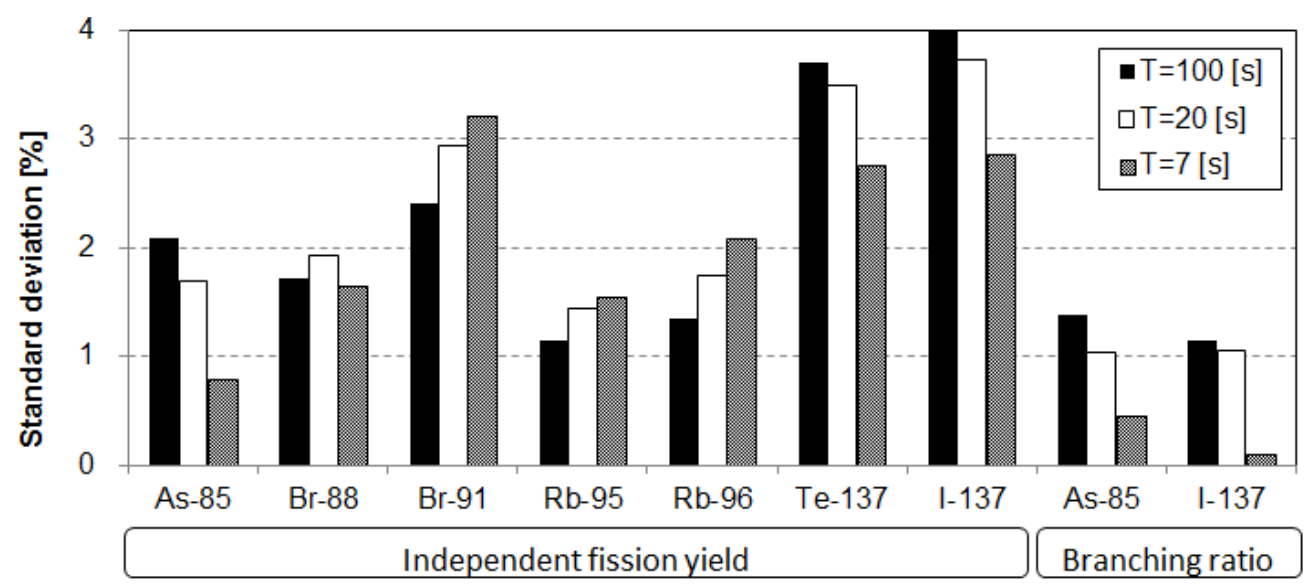

Figure 10 Nuclide-wise and Nuclear data-wise uncertainties of reactor stable period in the uranium-238 fast fission case

\section{G.Chiba:}

Sensitivity and uncertainty analysis for reactor stable period induced by positive reactivity using one-point adjoint kinetics equation 